\title{
Article \\ MiR408-SmLAC3 Module Participates in Salvianolic Acid B Synthesis in Salvia miltiorrhiza
}

\author{
Haolan Zou, Xiaorong Guo, Rao Yang, Shengsong Wang, Lin Li, Junfeng Niu, Donghao Wang (D) \\ and Xiaoyan Cao*(i)
}

Citation: Zou, H.; Guo, X.; Yang, R.; Wang, S.; Li, L.; Niu, J.; Wang, D.; Cao, X. MiR408-SmLAC3 Module Participates in Salvianolic Acid B Synthesis in Salvia miltiorrhiza. Int. J. Mol. Sci. 2021, 22, 7541. https:// doi.org/10.3390/ijms22147541

Academic Editor:

David Arráez-Román

Received: 17 May 2021

Accepted: 8 July 2021

Published: 14 July 2021

Publisher's Note: MDPI stays neutral with regard to jurisdictional claims in published maps and institutional affiliations.

Copyright: (C) 2021 by the authors. Licensee MDPI, Basel, Switzerland. This article is an open access article distributed under the terms and conditions of the Creative Commons Attribution (CC BY) license (https:/ / creativecommons.org/licenses/by/ $4.0 /)$.
Key Laboratory of the Ministry of Education for Medicinal Resources and Natural Pharmaceutical Chemistry, National Engineering Laboratory for Resource Development of Endangered Crude Drugs in Northwest of China, Shaanxi Normal University, Xi'an 710062, China; zhl2019@snnu.edu.cn (H.Z.); guoxiaorong@snnu.edu.cn (X.G.); yangrao@snnu.edu.cn (R.Y.); shengsongwang@snnu.edu.cn (S.W.); shidalilin@snnu.edu.cn (L.L.); niujunfeng@snnu.edu.cn (J.N.); wangdonghao@snnu.edu.cn (D.W.)

* Correspondence: caoxiaoyan@snnu.edu.cn

\begin{abstract}
MicroRNAs (miRNAs) are important regulators of gene expression involved in plant development and abiotic stress responses. Recently, miRNAs have also been reported to be engaged in the regulation of secondary plant metabolism. However, there are few functional studies of miRNAs in medicinal plants. For this study, we obtained Sm-miR408 interference lines to investigate the function of Sm-miR408 in a medicinal model plant (Salvia miltiorrhiza). It was found that inhibiting the expression of $S m$-miR408 could increase the content of salvianolic acid B and rosmarinic acid in the roots. The SmLAC3 and Sm-miR408 expression patterns were analyzed by qRT-PCR. A 5' RLM-RACE assay confirmed that $S m$-miR408 targets and negatively regulates SmLAC3. Moreover, the overexpression of SmLAC3 in S. miltiorrhiza promoted the accumulation of salvianolic acids in the roots. Furthermore, the lignin content of the roots in overexpressed SmLAC3 lines was decreased. Taken together, these findings indicated that $S m$-miR408 modulates the accumulation of phenolic acids in S. miltiorrhiza by targeting SmLAC3 expression levels.
\end{abstract}

Keywords: Sm-miR408; SmLAC3; salvianolic acid B; Salvia miltiorrhiza

\section{Introduction}

MicroRNAs (miRNAs) comprise a type of non-coding small RNA (typically 20-24 nucleotides in length) [1] that regulate the expression of target genes at the post-transcriptional level in animals and plants [2]. MiRNA can directly cleave target gene sequences when it shows a high degree of sequence complementarity to its target mRNA [3,4]. In the case of incomplete complementation, miRNA specifically binds to the 3 ' untranslated region of the target mRNA to inhibit translation [5]. With the further study of plant miRNAs, researchers have found that this class of regulatory molecule exhibits spatiotemporal and tissue specificity while playing essential roles in plant growth and development and responds to environment stimuli [6]. Plant miRNAs have been demonstrated to be involved in the regulation of seed development [7], leaf morphogenesis [8,9] as well as floral and root development [10-12]. Their transcription can also be regulated to further alter the expression of their target genes in response to drought [13], oxidative stress [14], copper deficiency [15], and other stressors [16].

The targets of plant miRNAs also contain genes that encode transcription factors to regulate the accumulation of secondary metabolites, which suggests that miRNAs participate in the regulation of secondary plant metabolism. In Arabidopsis, the miR163 target genes are members of the methyltransferase gene family, which is involved in the biosynthesis of plant secondary metabolites. They affect the conversion of farnesoic acid to methyl farnesoate, which perturbs insect growth and development by negatively regulating the expression of farnesoic acid methyltransferase [17]. MiR156-targeted SPLs 
can negatively regulate anthocyanin biosynthesis through the disruption of the MYBbHLH-WD40 transcriptional activation complex, which activates anthocyanin biosynthetic genes [18]. The overexpression of miR8154 and miR5298b in the Taxus cell line results in the upregulation of some key taxol genes, phenylpropane, and flavonoid biosynthesis such as taxadiene synthase, phenylalanine ammonia lyase, and chalcone synthase, which indicates that these miRNAs have some influence on secondary metabolism [19].

The miR408 family targets genes that encode several small blue proteins and laccase (a group of blue oxidases), both of which are blue copper proteins [20,21]. MiR408 maintains the stability of the internal environment by regulating the concentration of copper ions that participate in processes such as photosynthesis and cell wall lignification. Previous studies have shown that the expression of miR408 is not only affected by copper ion concentrations, but also by abiotic stress, which suggests that miR408 plays a certain role in the responses of plants to abiotic stresses [22-24]. Moreover, miR408 is involved in the regulation of secondary metabolism. The anthocyanin content of the transgenic seedlings overexpressing miR408 was 7-8 times that of wild type Arabidopsis [25]; however, the regulatory mechanism is unclear. Whether miR408 participates in the regulation of secondary metabolism in other plants, particularly in medicinal plants, is unknown. Although we investigated the functions of miR408 from Salvia miltiorrhiza (a model medicinal plant) by overexpressing Sm-MIR408 in Nicotiana benthamiana and found that it positively influences responses to salt tolerance [26], the functions of miR408 in S. miltiorrhiza have not been reported as yet.

Laccase (EC 1.10.3.2) is a type of blue multicopper oxidase that contains four copper atoms and has been found to be widespread in plants, bacteria, fungi, and insects [27]. Research over the years has revealed that laccase in plants is associated with lignin polymerization [28] and flavonoid biosynthesis [29]. For instance, a knockout of AtLAC11 along with either AtLAC4 or AtLAC17 and a lac11lac4lac17 triple mutant significantly reduced the lignin content [30]. AtLAC15 mutants accumulated more epicatechin monomers and soluble proanthocyanidins than wild-type seeds, suggesting that $L A C 15$ may contribute to the oxidative polymerization of flavonoids [31]. In exploring the function of cotton LACs, researchers found that GaLAC1 promotes the conversion of sinapic acid to a monolactonetype dimer [32]. Nevertheless, the biochemical or physiological functions of laccases in plants remain largely unclear, and related reports are rare.

Salvianolic acid B (SalB) is one of the main active ingredients of S. miltiorrhiza and is considered a dimer of rosmarinic acid (RA). The biosynthetic pathway of RA in plants has been demonstrated (Figure 1); however, how RA is converted into SalB is still unknown. It has been speculated that laccases in S. miltiorrhiza might catalyze RA to SalB [33,34]. In the present study, the function of $S m$-miR408 was investigated by knocking down its expression in S. miltiorrhiza. SmLAC3 was verified as the authentic target of Sm-miR408 using $5^{\prime}$ RLM-RACE. The enhanced expression of SmLAC3 was achieved through gain-of-function analysis. The contents of SalB and RA were increased in both miR408-suppressed and $S m L A C 3$-overexpressed transgenic lines. The results suggested that $S m$-miR408 regulated the biosynthesis of SalB using its target gene SmLAC3. To the best of our knowledge, this is the first functional study of miRNA in S. miltiorrhiza and the first report of miRNA being involved in the regulation of the active ingredients in a medicinal plant. 


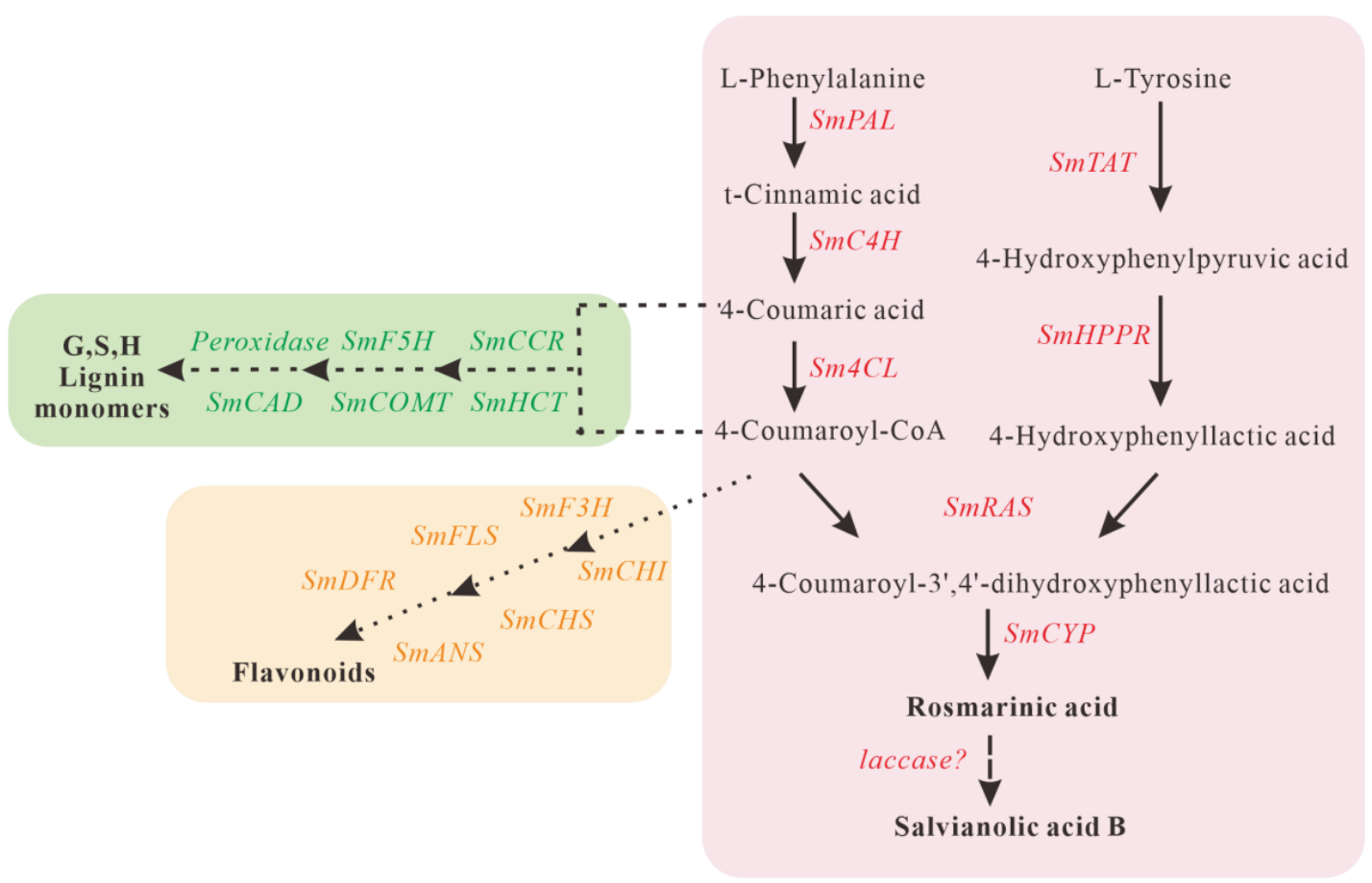

Figure 1. The proposed biosynthetic pathways for phenolic acids, flavonoids, and lignin in Salvia miltiorrhiza. PAL, phenylalanine ammonia lyase; $C 4 H$, cinnamate 4-hydroxylase; $4 C L$, hydroxycinnamate-CoA ligase; $T A T$, tyrosine aminotransferase; $H P P R$, hydroxyl phenylpyruvate reductase; $R A S$, rosmarinic acid synthase; CYP, cytochrome P450 enzymes; $C H I$, chalcone isomerase; $F 3 H$, flavanone 3-hydroxylase; $C H S$, chalcone synthase; FLS, flavonol synthase; DFR, dihydroflavonol 4-reductase; ANS, anthocyanin synthase; HCT, hydroxyl cinnamoyl transferase; CCR, cinnamoyl-CoA reductase; COMT, caffeic acid O-methyltransferase; $F 5 H$, ferulate 5-hydroxylase; $C A D$, cinnamyl alcohol dehydrogenase. Solid lines indicate the step is specific. Dashed lines indicate the step is uncertain or multiple enzymatic steps are represented.

\section{Results}

\subsection{MiR408 Negatively Regulates the Synthesis of SalB and RA in S. miltiorrhiza}

To investigate the function of miR408 in S. miltiorrhiza, we obtained miR408-suppressed transgenic plantlets (amiR408) via Agrobacterium-mediated transformation. After selective culturing on an herbicide medium, three transgenic lines (amiR408-3, amiR408-4, and amiR408-5) were used to detect the expression of miR408. Quantitative reverse transcription PCR (qRT-PCR) results indicated that the expression levels of both the precursor and mature miR408 were significantly suppressed in the transgenic plants compared to that of the control (Figure 2). In comparison to the control, the Sm-miR408 interference lines did not show an obvious phenotype change (Figure 2). Two transgenic lines, amiR408-3 and amiR408-4, were used for the following experiments.

To determine whether knocking down the expression of miR408 would affect the synthesis of phenolic acids in S. miltiorrhiza, we tested the concentrations of RA and SalB via high performance liquid chromatography (HPLC). The results showed that both RA and SalB were significantly increased compared to those of the untransformed control. As shown in Figure 3A, the quantity of RA was 1.4 and 1.82 times higher in amiR408-3 and amiR408-4, respectively, while the content of SalB was approximately 2.52 times and 4.11 times higher than those of the control (Figure 3B). 
A

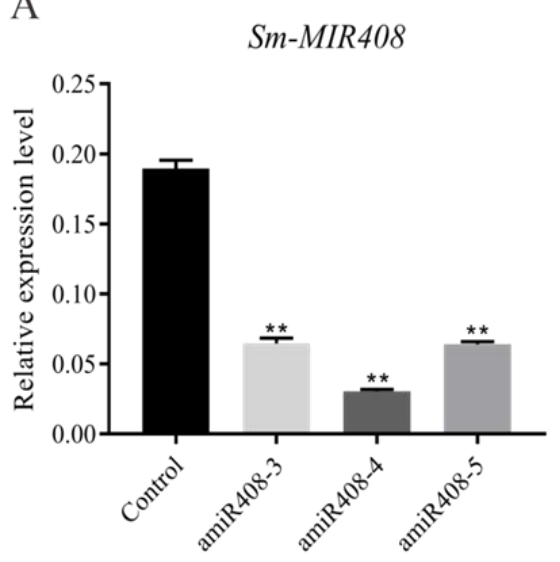

$\mathrm{B}$

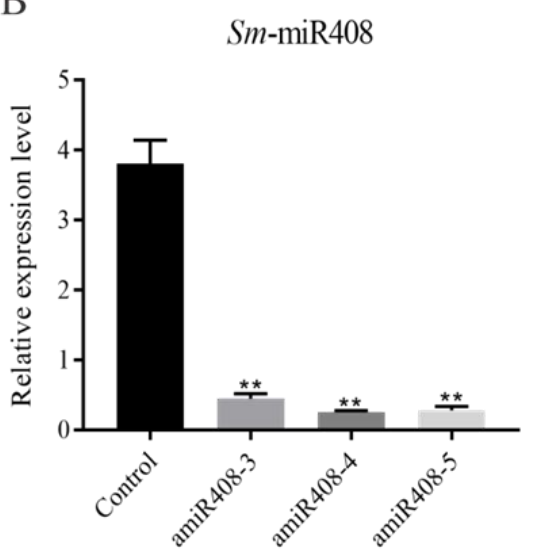

$\mathrm{C}$

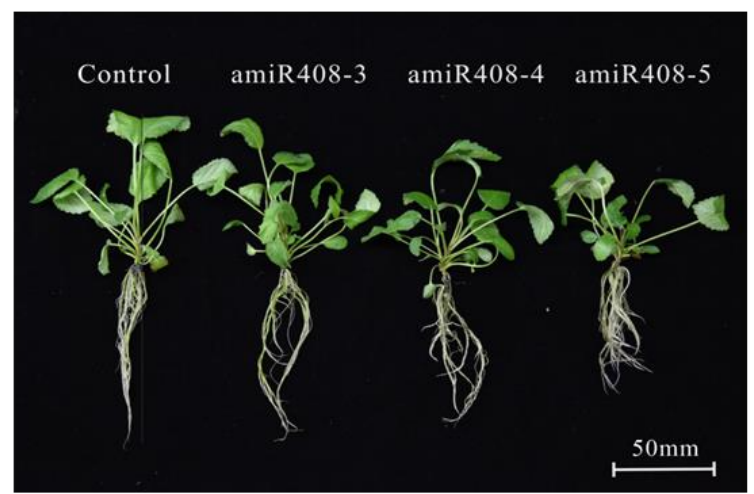

Figure 2. Identification and phenotype of the miR408-suppressed transgenic Salvia miltiorrhiza. (A) Expression level of the precursor miR408. (B) Expression level of mature miR408. (C) Phenotype of the miR408-suppressed transgenic lines (amiR408-3, amiR408-4, and amiR408-5) and the control. All data are means of three independent experiments, with error bars representing SD. Significant differences in comparison with the control were determined by Dunnett's multiple comparison test (** $p<0.01)$.

We also undertook objective of detecting the total phenolics and total flavonoids, both of which share an upstream core phenylpropanoid metabolism with SalB. The results revealed that the levels of total phenolics and total flavonoids were significantly higher in the transgenic lines than in the control (Figure 3C,D). Compared to the control, the content of total phenolics increased by 1.42 times and 1.61 times in amiR408-3 and amiR408-4, respectively, and the total flavonoids increased by 1.59 times and 2.08 times, respectively. In addition, we measured the anthocyanin content in these lines and found that its level in the transgenic lines was lower than in the control (Figure 3E).

The above results demonstrated that inhibiting the expression of miR408 leads to higher phenolic acid levels. Furthermore, we examined the transcript levels of six genes in the RA synthetic pathway and the results indicated that most of those genes, including SmTAT1, SmPAL2, SmC4H1, Sm4CL2, and SmHPPR1, were significantly induced in the amiR408 lines (Figure 4). In particular, the transcript level of TAT1 was increased by 4.2and 3.5 times in amiR408-3 and amiR408-4, respectively. All of these findings demonstrated that knocking down miR408 induces the expression of enzyme genes in the phenolic acid biosynthetic pathway, leading to the enhancement of RA and SalB content. 


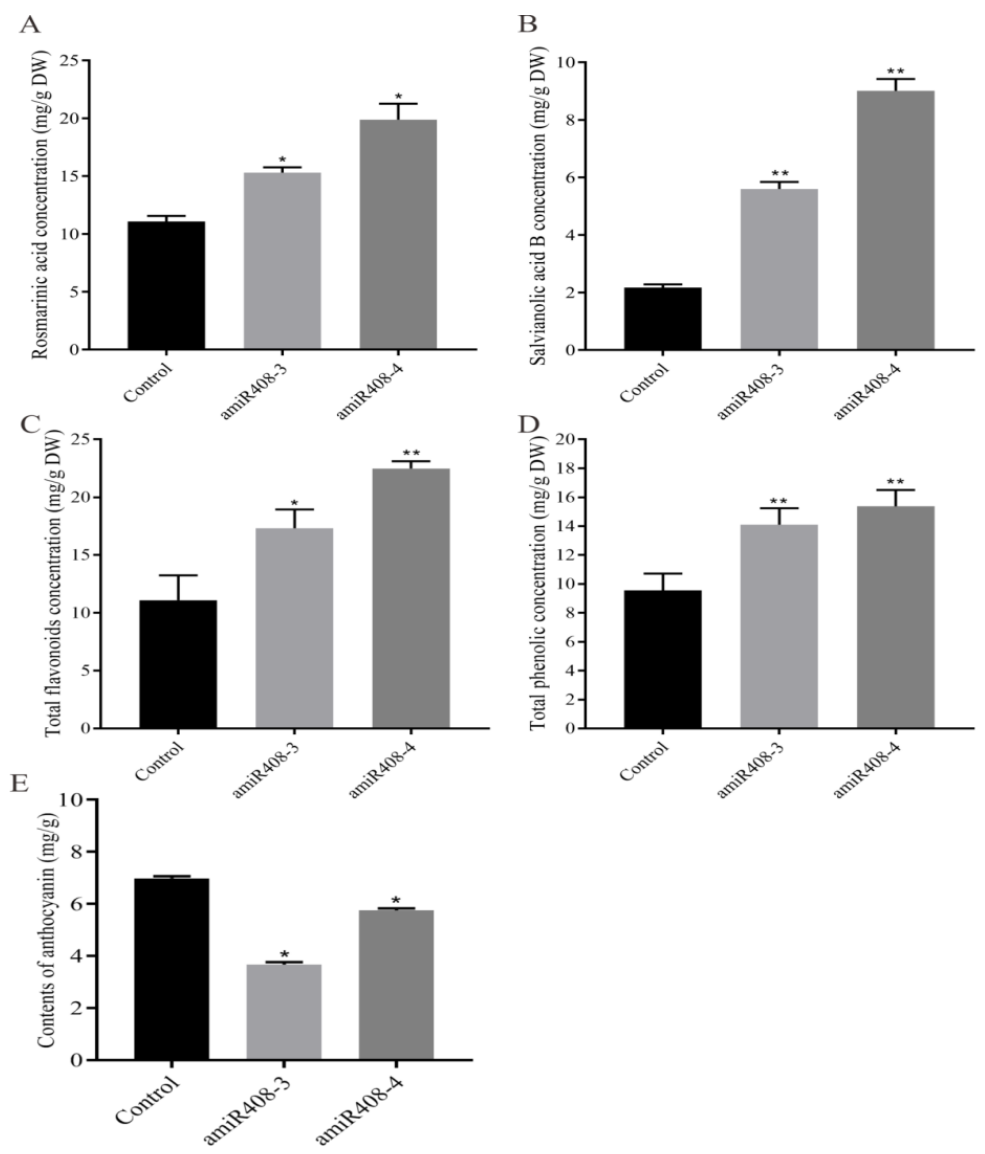

Figure 3. Detection of rosmarinic acid (A), salvianolic acid B (B), total flavonoids (C), total phenolic (D), and anthocyanin (E) content in transgenic (amiR408) and control lines. All data represent mean \pm SD of three biological replicates. Significant differences in comparison with the control were assessed with Dunnett's multiple comparison test $\left({ }^{* *} p<0.01 ;{ }^{*} p<0.05\right)$.
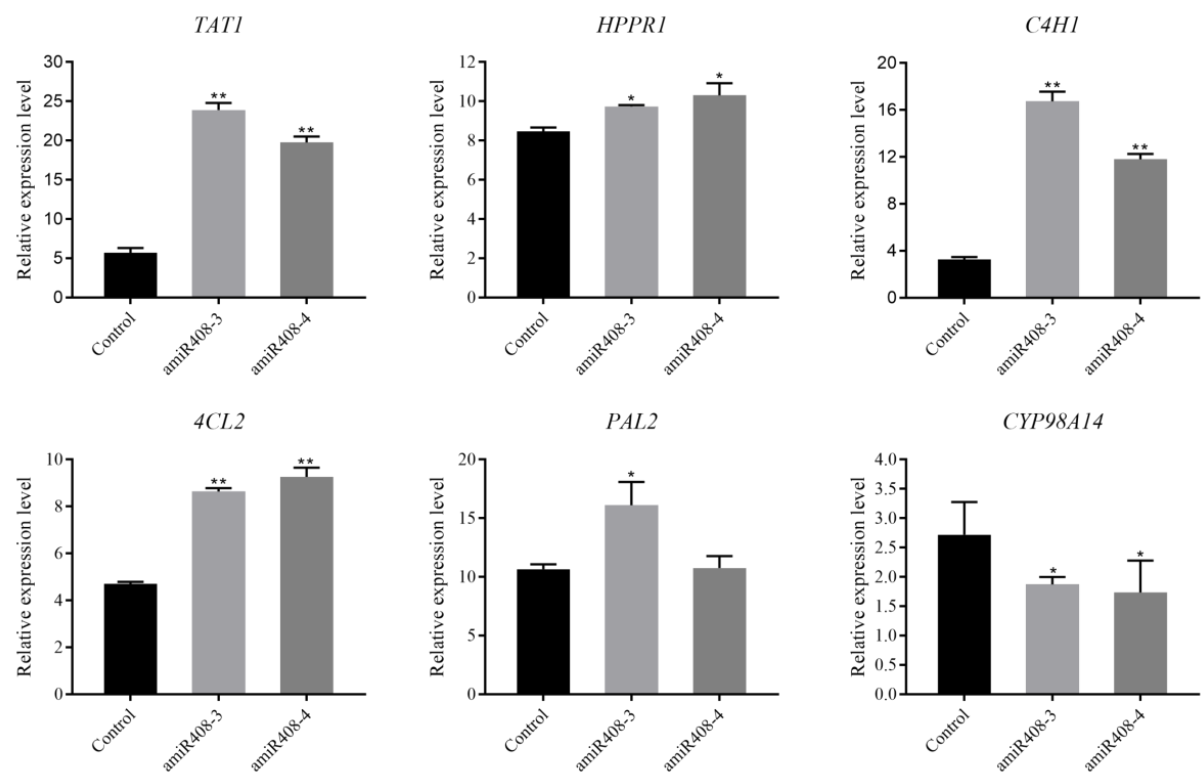

Figure 4. Relative expression levels of the key enzyme genes involved in phenolic acid biosynthetic pathway in the transgenic (amiR408) and control lines. All data are means of three independent experiments, with error bars representing SD. Significant differences in comparison with the control were determined by Dunnett's multiple comparison test $\left({ }^{* *} p<0.01 ;^{*} p<0.05\right)$. 


\subsection{SmLAC3 Is the Target of Sm-miR408}

It is well known that miRNAs exert their functions by inhibiting the expression of target genes, and miRNAs need to be strictly complementary with their target genes in order to cleave at the pairing sites [35,36]. To reveal the target of Sm-miR408, we predicted its potential targets through the website psRNATarget (http://plantgrn.noble.org/psR NATarget/, accessed in 12 May 2018) [37]. Under relatively strict parameters, SmLAC3, SmCYP72A327, and SmBRI-like3, which encode laccase, the cytochrome P450 enzyme, and the putative receptor-like protein kinase brassinosteroid insensitive 3, respectively, were predicted as the putative targets of $S m$-miR408.

To validate the targets of $S m$-miR408, we analyzed the transcription levels of SmmiR408 and its putative target genes using qRT-PCR. First, their transcripts were detected in the control and amiR408 lines. As shown in Figure 5A, compared to the control, the expression levels of the three putative targets were significantly up-regulated in the amiR408 lines. The abundance of miR408 is negatively regulated by the availability of copper [15] and has been annotated in more than 20 plant species [38]. Consequently, we detected the expression levels of Sm-miR408 and its putative targets in S. miltiorrhiza under normal and copper starvation conditions (Figure 5B). The results indicated that Sm-miR408 accumulated to a higher level under copper-deficient conditions. Among the three putative target genes, compared to the normal condition, only SmLAC3 was significantly down-regulated under copper starvation conditions. Hence, qRT-PCR analysis revealed that $S m L A C 3$ was the most prospective target of $\mathrm{Sm}$-miR408.

A
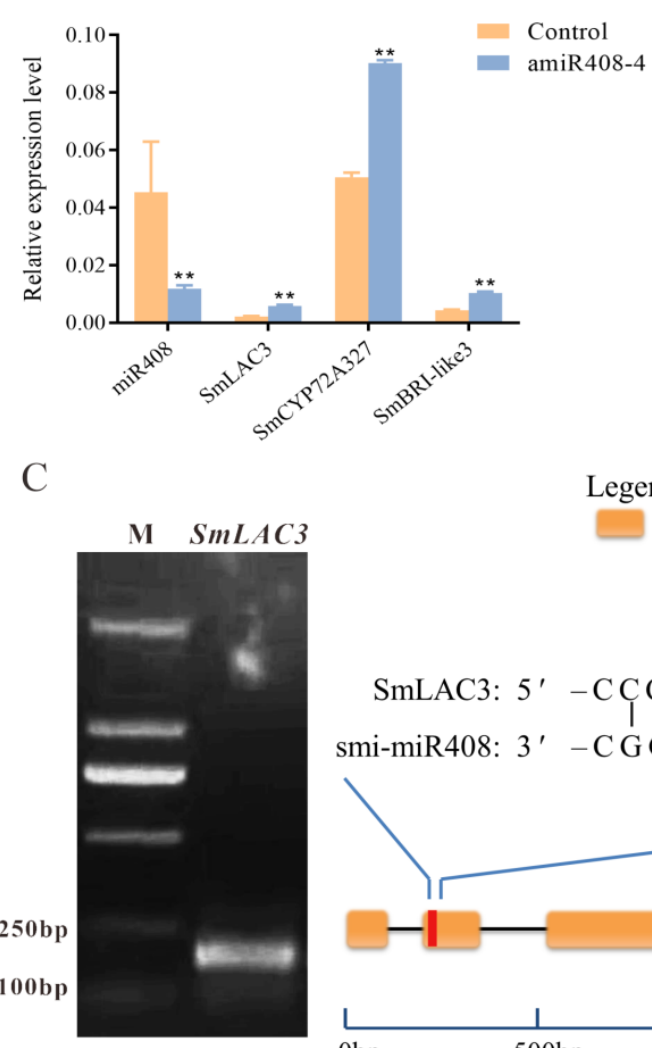

$\mathrm{B}$

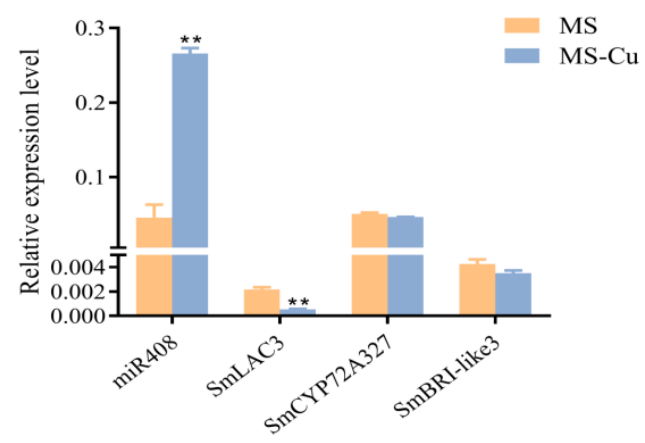

Legend:

Exon

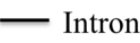

$$
5 / 8
$$

SmLAC3: 5' -CCCAGUGAAGAGGCUGUGCAA- 3' smi-miR408: 3' -CGGUCCCUUCUCCGUCACGUA- 5'

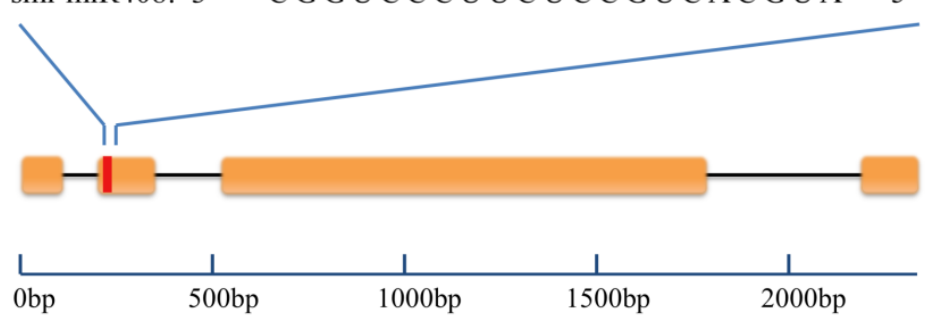

Figure 5. Validation of Sm-miR408 target genes. (A) Relative expression levels of the Sm-miR408 putative target genes in amiR408 lines compared to control lines. (B) Relative expression levels of the Sm-miR408 putative target genes under normal and copper starvation conditions. (C) Sm-miR408 cleavage site in SmLAC3 mRNA validated by 5' RLM-RACE. For RT-qPCR, all data represent the means of three biological replicates, with error bars indicating SD. Significant differences were determined by Student's $t$-test $\left({ }^{* *} p<0.01\right)$. 
The 5' RNA ligase-mediated rapid amplification of cDNA ends (5' RLM-RACE) is a PCR-based technique that has been widely employed to validate the cleavage sites of target genes using miRNA $[39,40]$. Here, we performed 5'RLM-RACE to detect whether $\mathrm{Sm}$-miR408 cleaved its target genes. The results indicated that of the eight randomly selected clones, five possessed the $5^{\prime}$ ends of the cleaved fragments at the same site as the $S m L A C 3$. The cleavage site for $S m L A C 3$ resided between the 11th and 12th nucleotides of smi-miR408 (Figure 5C); however, SmCYP72A327 and SmBRI-like3 were not cleaved by miR408. These results indicated that SmLAC3 was an authentic target gene of miR408, and its expression level was contingent on the miR408-directed cleavage modes of the miR408.

\subsection{Expression Profiles of SmLAC3 in S. miltiorrhiza}

We investigated the spatial expression patterns of $S m L A C 3$ in various tissues using qRT-PCR analysis. As shown in Figure 6A, SmLAC3 was expressed in all of the tested tissues, and it exhibited the highest level in the calyxes, followed by the stems and stamen, with the lowest transcript level in the corollas and leaves.

A

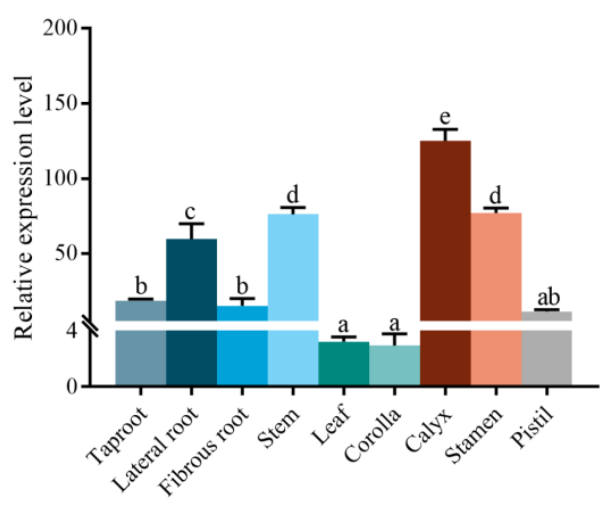

B

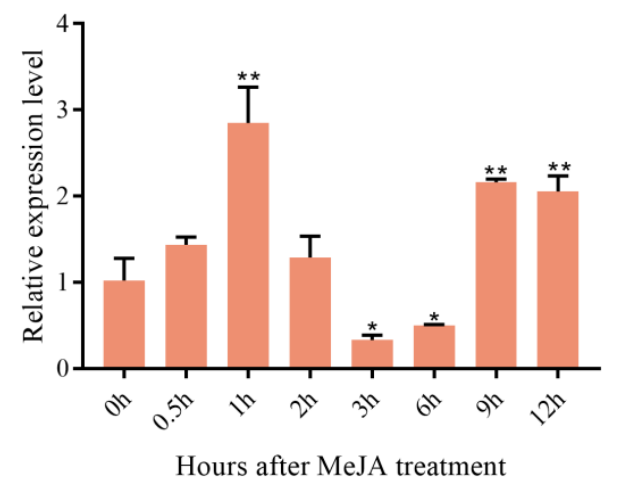

Figure 6. Expression patterns of SmLAC3. (A) qRT-PCR analysis of SmLAC3 expression in various S. miltiorrhiza tissues. Different lowercase letters indicate significant differences at $p<0.05$ by one-way ANOVA with Tukey's multiple comparisons test. (B) SmLAC3 expression in response to a $100 \mu \mathrm{M}$ MeJA treatment. All data represent the means of three biological replicates, with error bars indicating SD. Significant differences were determined by Dunnett's multiple comparison test $\left({ }^{* *} p<0.01 ;{ }^{*} p<0.05\right)$.

Most genes in phenolic acid biosynthesis can be induced by methyl jasmonate (MeJA), which leads to the accumulation of SalB [41,42]. We detected the expression profile of SmLAC3 under the condition of MeJA treatment. The results indicated that SmLAC3 responded to the MeJA treatment (Figure $6 \mathrm{~B}$ ), and significant expression was induced at $1 \mathrm{~h}, 9 \mathrm{~h}$, and $12 \mathrm{~h}$ following the MeJA treatment, but was significantly suppressed at $3 \mathrm{~h}$ and $6 \mathrm{~h}$. These fluctuating changes showed that the expression profile of SmLAC3 was complicated when treated by MeJA.

\subsection{Overexpression of SmLAC3 Promotes the Biosynthesis of SalB and RA in S. miltiorrhiza}

To elucidate whether $S m L A C 3$ affects the synthesis of SalB and RA, we generated transgenic plants that overexpressed SmLAC3 (SmLAC3-OE). We detected the SmLAC3 expression level in these OE lines using qRT-PCR, with the results showing that the transcripts of $S m L A C 3$ in all of the transgenic lines were significantly higher than those in the control (Figure 7A). Four SmLAC3-OE lines with higher expression levels (OE-2, OE-10, OE-15, and OE-17) were selected for subsequent experiments. As anticipated, a significant increase in the concentrations of both RA and SalB was observed in the roots of the SmLAC3-OE lines. 


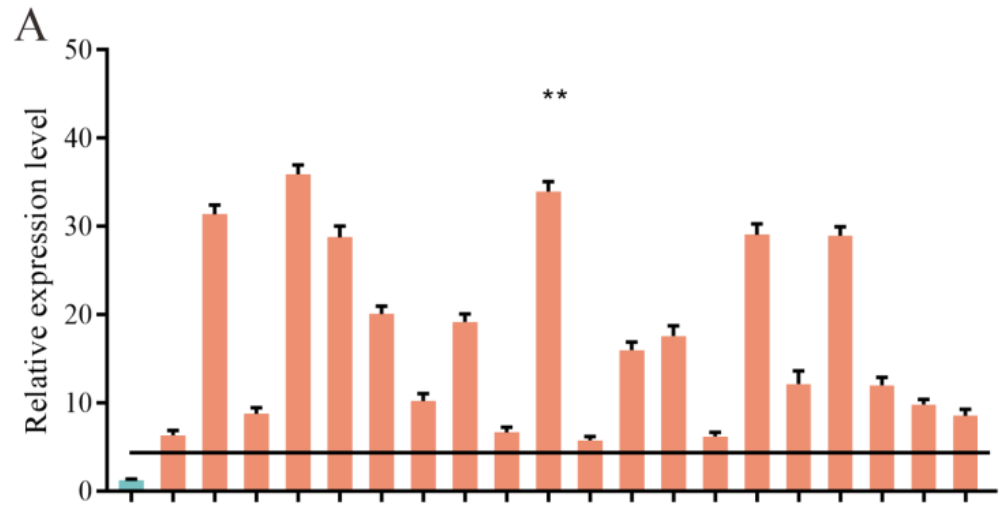
co
D

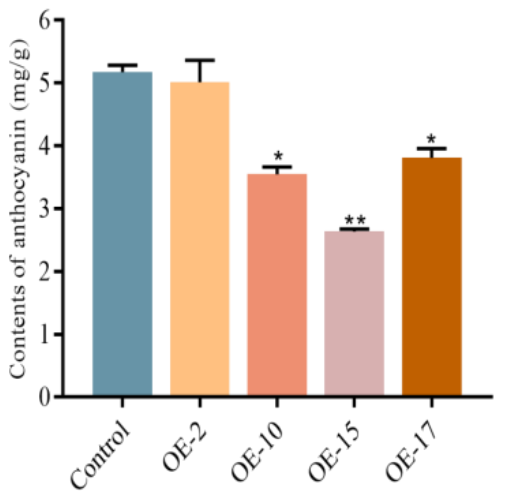

$\mathrm{B}$

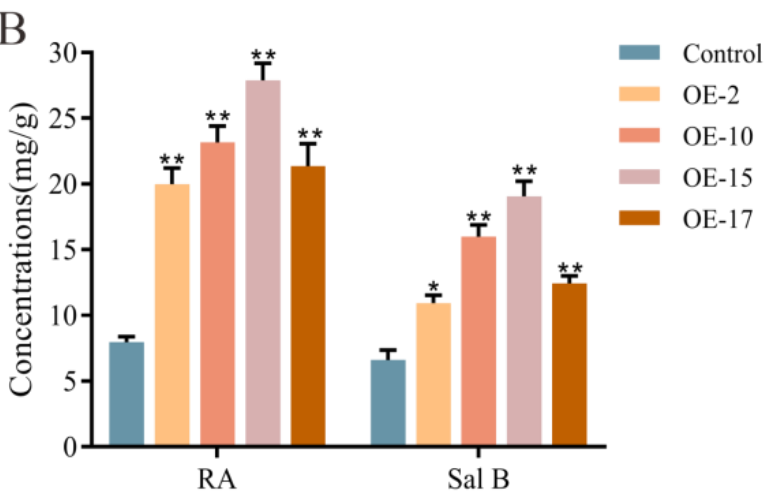

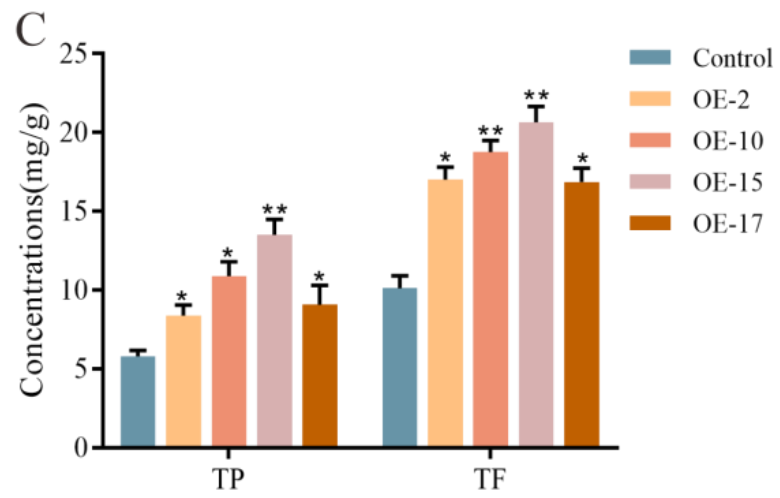

E

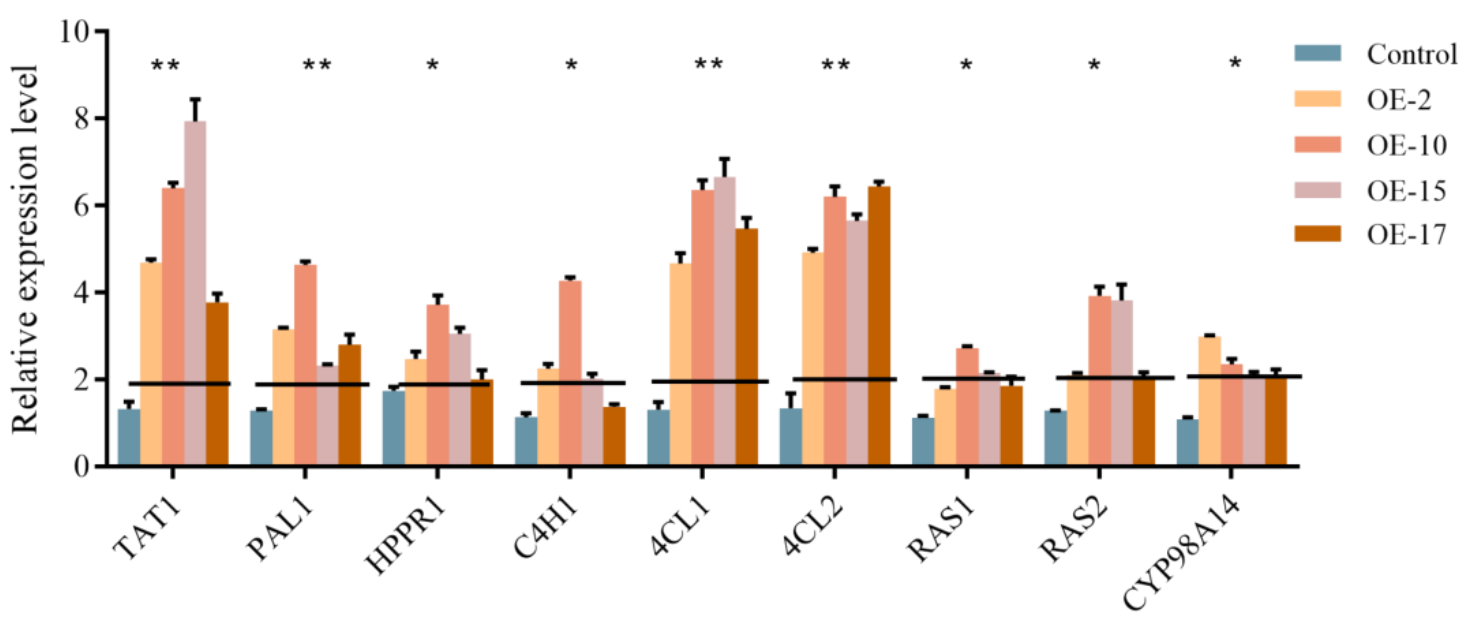

Figure 7. Effects of overexpressed $S m L A C 3$ on the phenolic acid biosynthesis pathway. (A) qRT-PCR analysis of $S m L A C 3$ expression in transgenic and control lines. (B) Concentrations of RA and SalB in the roots of SmLAC3-OE and control lines. (C) Concentrations of TP and TF in the roots of SmLAC3-OE and control lines. (D) Detection of anthocyanin content in SmLAC3-OE and control lines. (E) Relative expression levels of genes involved in the SmLAC3-OE phenolic acid pathway and control lines. All data are the means of three independent experiments, with error bars representing SD. Significant differences in comparison with the control were determined by Dunnett's multiple comparison test $\left({ }^{*} p<0.01 ;^{*} p<0.05\right)$. 
The quantities of RA were 2.44 times, 2.93 times, 3.48times, and 2.62times higher in OE-2, OE-10, OE-15, and OE-17, respectively, than that of the control, while the amount of SalB was approximately 1.82 times, 2.79times, 3.42times, and 2.13times higher, respectively, than that of the control (Figure 7B). Moreover, the total phenolic and total flavonoid concentrations of the SmLAC3-OE lines were significantly increased when compared to the control. Between the four lines, OE-15 showed the highest degree of change with the levels of total phenolics and total flavonoids being 2.50 times and 2.06 times higher, respectively (Figure 7C). We also detected the content of anthocyanin in two SmLAC3-OE lines, and the results showed that its level was lower than that in the control lines (Figure 7D).

We further examined the transcript levels of nine enzyme genes of the biosynthetic pathway for phenolic acids in the SmLAC3-OE lines using qRT-PCR, with the results shown in Figure 7E. These genes were all significantly up-regulated in the SmLAC3-OE lines. Among the tested genes, the expression levels of SmTAT1, Sm4CL1, and Sm4CL2 exhibited more pronounced changes. In OE-2, OE-10, OE-15, and OE-17, the SmTAT1 expression levels were up-regulated by 4.53 times, 6.35 times, 7.92 times, and 3.31 times, respectively, when compared to the empty vector control lines; Sm4CL1 was up-regulated by 4.81 times, 6.13 times, 6.38 times, and 5.42 times, respectively; and Sm4CL2 was upregulated by 5.09 times, 6.21 times, 5.36 times, and 6.32 times, respectively. These data suggest that the overexpression of SmLAC3 in transgenic S. miltiorrhiza promoted the transcription of genes for the biosynthesis of phenolic acids and increased the concentrations of RA and SalB.

\subsection{Overexpressing SmLAC3 Decreases the Lignin Content in the Roots}

Previous studies have shown that the laccase of plants can promote the synthesis of lignin $[43,44]$. Lignin and phenolic acids share a common upstream phenylpropanoid pathway [34,45]. We were curious as to the changes in the lignin content in the roots of SmLAC3-OE lines; thus, the safranin staining method was used to explore whether the hyper-accumulation of SmLAC3 alters the lignification of cell walls in the roots of S. miltiorrhiza.

As shown in Figure 8, compared to the control, the staining of the SmLAC3-OE lines was lighter and less vivid, which indicated that less lignin was deposited in the roots of the SmLAC3-OE lines. We further determined the lignin content in the SmLAC3-OE lines and the control, and the result showed that the lignin content in the roots of the SmLAC3-OE lines was lower than that of the control (Table 1), which was consistent with the staining results. We speculated that the decrease of lignin in the SmLAC3-OE lines might be caused by a reduction of metabolic flux into the lignin biosynthesis branch due to the significant increase of phenolic acid biosynthesis.

Table 1. Lignin contents in the roots of the SmLAC3-OE lines and the control.

\begin{tabular}{ccc}
\hline \multirow{2}{*}{ Line } & \multicolumn{2}{c}{ Lignin $(\mathbf{m g} / \mathbf{g}$ DW) } \\
\cline { 2 - 3 } & Klason Lignin & Acid-Soluble Lignin \\
\hline Control & $72.13 \pm 2.31$ & $0.53 \pm 0.01$ \\
OE-2 & $63.99 \pm 3.26$ & $0.47 \pm 0.02$ \\
OE-10 & $59.62 \pm 3.05$ & $0.45 \pm 0.02$ \\
OE-15 & $50.96 \pm 1.71^{*}$ & $0.38 \pm 0.01^{*}$ \\
OE-17 & $52.48 \pm 2.14^{*}$ & $0.41 \pm 0.03^{*}$ \\
\hline
\end{tabular}

$\overline{\text { Data are mean values and standard errors (means } \pm \text { SDs). Significant differences were determined by one-way }}$ ANOVA test relative to the control value $\left.{ }^{*} p<0.05\right)$. 
A

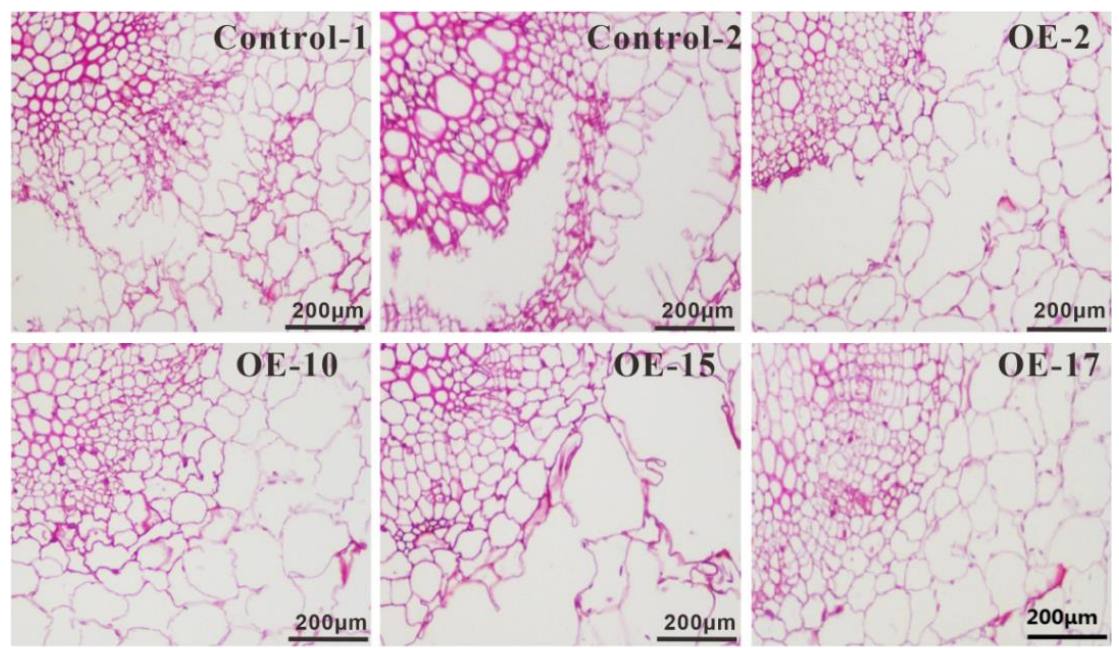

B

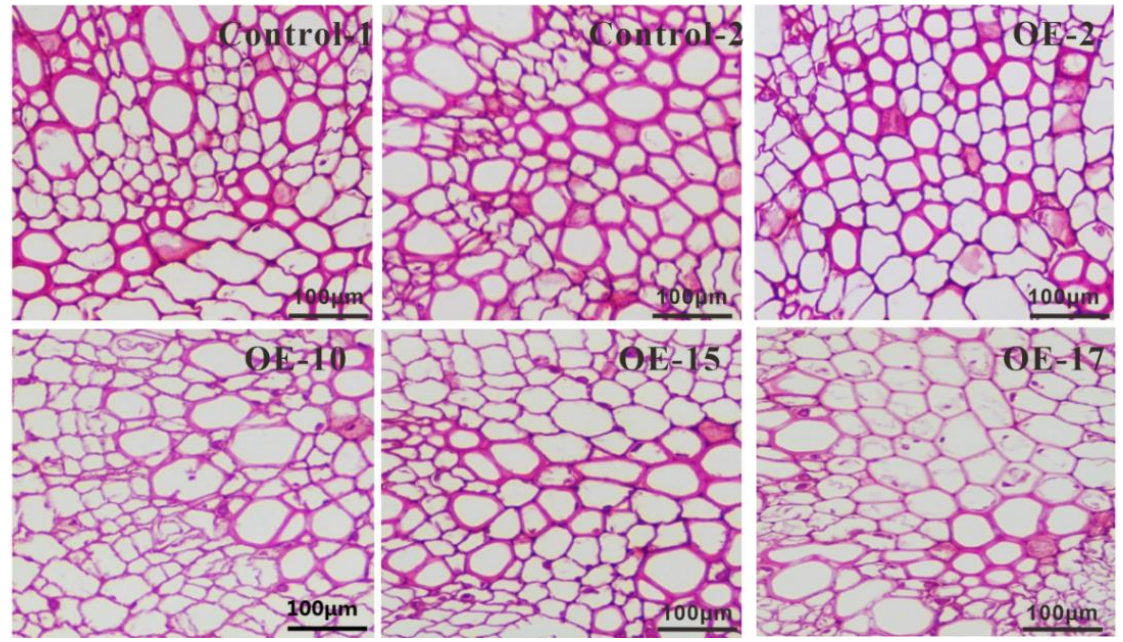

Figure 8. Histochemical stain of lignin in the roots of SmLAC3-OE and control lines. (A) Lignin staining in the roots of the control and transgenic lines. Scale bar denotes $200 \mu \mathrm{m}$. (B) Magnified views of images in A. Scale bar denotes $100 \mu \mathrm{m}$.

\section{Discussion}

\subsection{MiR408 Has Multiple Functions in Plants}

MiR408 is a class of highly conserved miRNA in plants that is composed of $21 \mathrm{nu}-$ cleotides [46]. Since its initial discovery in Arabidopsis thaliana, miR408 has been found in a variety of plants. Current research on miR408 in plants has focused on its important roles in plant growth and development as well as in responses to abiotic stress. In A. thaliana, the upregulation of miR408 enhanced plant tolerance to cold, salinity, and oxidative stress and delayed plant senescence [21]. The overexpression of miR408 in rice led to a higher pollen germination rate [47]. Overexpressed miR408 also translated to an improvement in photosynthetic performance, a higher rate of vegetative growth, and increased seed yields in A. thaliana, Oryza sativa, and Nicotiana tabacum [23]; however, the overexpression of miR408 in Ipomoea batatas resulted in a semi-dwarf phenotype [48]. In the present study, miR408-suppressed S. miltiorrhiza showed no obvious phenotype changes.

Furthermore, miR408 was reported to be involved in the regulation of secondary metabolism, where overexpressed miR408 in Arabidopsis significantly promoted the accumulation of anthocyanin [25]. Here, we measured the levels of total phenolics, total flavonoids, and anthocyanin in the miR408-suppressed transgenic S. miltiorrhiza (Figure 3). It was found that the concentrations of total flavonoids and total phenolics increased, 
while the anthocyanin content in the miR408 interference lines decreased in contrast to the control, which was consistent with the results in A. thaliana (Figure 3C).

As a model medicinal plant, $S$. miltiorrhiza contains several representative active ingredients, such as SalB and RA. We determined their contents in miR408 interference lines and found that both SalB and RA were significantly increased (Figure 3A). These results suggested miR408 affected not only the accumulation of anthocyanin in S. miltiorrhiza, but also the synthesis of salvianolic acids. To the best of our knowledge, this is the first report to articulate the functions of miRNA in S. miltiorrhiza.

\subsection{Function of Laccase in S. miltiorrhiza}

Laccase is extensively distributed across various organisms in nature, encompassing fungi, bacteria, plants, and insects. Although the structures of laccases are similar in different species, their functions are not identical [49-51]. To date, research on laccase has mainly focused on fungal laccase, with few studies on the functions of laccase in plants. Most of laccases in plants are involved in lignification. Regulation of lignin synthesis plays an important role in maintaining the structural integrity of cell wall and improving plant defense [52]. Moreover, in some bioenergy crops, the change of lignin composition will affect the biosaccharification rate, affecting the economic value of the crops [52]. In Gossypium hirsutum, both GhLAC1 overexpression and RNAi lines exhibited enhanced resistance to Verticillium dahliae and cotton bollworm. Interestingly, however, overexpressed GhLAC1 also increased resistance to cotton aphids, while down-regulated GhLAC1 showed the opposite. This revealed the critical role of GhLAC1 in the phenylpropanoid pathway and in the rebalancing of jasmonic acid and salicylic acid [53].

Di et al. (2013) speculated that laccase in S. miltiorrhiza might catalyze RA to SalB [34]. Based on the genome sequence of S. miltiorrhiza, Li et al. (2019a) identified 29 laccase candidates (SmLAC1-SmLAC29) that contained three signature Cu-oxidase domains [33]. The downregulation of SmLAC7 and SmLAC20 in the hairy roots of S. miltiorrhiza led to decreased levels of SalB and RA, while their overexpression led to increased levels [33]. In this study, we obtained SmLAC3 overexpressing transgenic S. miltiorrhiza lines and found that the SalB and RA contents were increased in these transgenic lines (Figure 7B). Although these results supported previous speculation that laccase might catalyze RA to SalB, direct in vitro evidence regarding the catalytic activity of the enzyme was lacking. We did successfully obtain purified recombinant SmLAC3 via the prokaryotic expression vector pGEX-4T-1 (data not shown) but failed to obtain the active protein. Efforts should be taken to ascertain the SmLAC3 substrate in the future.

In Arabidopsis, lignin synthesis was found to be blocked in the lac11 lac4 lac17 triple mutant, which suggested that these three laccases may be involved in lignin polymerization [30]. In the ornamental plant Cleome hassleriana, ChLAC8 was verified to have a promoting effect on the polymerization of C-lignin [54]. However, our results indicated that the overexpression of SmLAC3 decreased lignin synthesis in the roots of S. miltiorrhiza (Figure 8). Since lignin and phenolic acids share a common upstream phenylpropanoid pathway (Figure 1), we speculated that the decrease of lignin in the SmLAC3-OE lines might be caused by the reduction of metabolic flux into the lignin biosynthesis branch due to the significant increase of phenolic acid biosynthesis.

\subsection{SmLAC3 Is the Target of Sm-miR408}

Degradome sequencing and 5' RLM-RACE are widely used for verifying miRNA cleavage sites. MiR408 often targets genes encoding copper-binding proteins, such as laccase (LAC) and plantacyanin. In Arabidopsis, LAC3, LAC12, and LAC13 were predicted and validated by 5' RLM-RACE as target genes of miR408 [15]. Plantacyanin (TC116986) was identified to be the target of Mtr-miR408 in Medicago truncatula following 5' RLMRACE analysis [22]. Moreover, the study found that dlo-miR408-3p targeted DILAC12 in Dimocarpus longan Lour [55]. 
Li et al. (2019b) verified that three members (SmLAC3, SmLAC18, and SmLAC28) of the 65 LAC gene family in $S$. miltiorrhiza were the target of miR408 via degradome sequencing, and two members (SmLAC3 and SmLAC18) were simultaneously verified by a 5' RLMRACE experiment [56]. In the present study, qRT-PCR revealed that the transcript levels of Sm-miR408 and SmLAC3 showed an obvious opposite trend, and 5' RLM-RACE confirmed that SmLAC3 is an authentic target gene of miR408 (Figure 4). It must be mentioned that our SmLAC3 was named according to the 29 laccase members reported by Li et al. (2019a) and that the sequence of SmLAC3 was consistent with the SmLAC28 of the 65 members.

\section{Materials and Methods}

\subsection{Experimental Materials}

Seeds of S. miltiorrhiza were surface-sterilized, as described previously [57]. Sterile $S$. miltiorrhiza seedlings and transgenic $S$. miltiorrhiza were cultured on a MS basal medium under standard light and temperature growth conditions $\left(22{ }^{\circ} \mathrm{C}\right.$ light intensity $100 \mu \mathrm{mol} \mathrm{m}{ }^{-2} . \mathrm{s}^{-1}, 16 / 8 \mathrm{~h}$ light/dark cycle). All chemicals were purchased from Sigma Chemical Co. (St. Louis, USA). Solvents were of high-performance liquid chromatography (HPLC) grade. The standards (RA, SalB) used for HPLC were from the National Institute for the Control of Pharmaceutical and Biological Products (Beijing, China). The relevant primers are listed in Table 2.

Table 2. List of primers used for PCR.

\begin{tabular}{|c|c|}
\hline Primer & Oligo Sequence $5^{\prime}$ to $3^{\prime}$ \\
\hline amiR-MIR408-F & TGTATCTGTCTCGTCCCCGTCTCCAATGATGATCACATTCGTTATCTATTTTTTTGGAGACGGGTACGAGACAGA \\
\hline amiR-MIR408-R & AATGTCTGTCTCGTACCCGTCTCCAAAAAAATAGATAACGAATGTGATCATTGGAGACGGGGACGAGACAGA \\
\hline RT-SmUbiquitin-F & ACCCTCACGGGGAAGACCATC \\
\hline RT-SmUbiquitin-R & ACCACGGAGACGGAGGACAAG \\
\hline RT-MIR408-F & ACAGAAAATGGAGGCGAAGAAG \\
\hline RT-MIR408-R & GTCCCTAATCAGTGAGAGACACAGTAA \\
\hline RT-SmLAC3-F & TGTTGCTCCTTTGGATGCCT \\
\hline RT-SmLAC3-R & CCGTGATCGTGTTGTGGGTT \\
\hline RT-SmCYP72A327-F & GCTACCAACAAGGCAGAAGGAT \\
\hline RT-SmCYP72A327-R & ССТСTCTTCCCATCGCTTCC \\
\hline RT-SmBRI-like3-F & GAAGCAGTTCGCATTCGTGA \\
\hline RT-SmBRI-like3-R & GACCCGAGAGTTGATTGTAGGA \\
\hline SmLAC3-101 & GGATACATCCCATTCACCGTGATC \\
\hline SmLAC3-152 & ATAACCTTCACCACCAGAGTGTCG \\
\hline SmLAC3-217 & CCCATGCACTTCTCATTTGCCT \\
\hline SmLAC3-F & ATGGAGAAGAAGATGAGCTCTTTG \\
\hline SmLAC3-R & TCAACAAAGGGGAAGATCTGG \\
\hline RT-TAT1-F & CGAGCAGGGATGGGAGGTTG \\
\hline RT-TAT1-R & GCCTCTTGGCTGTCTCAGCA \\
\hline RT-PAL1-F & GATAGCGGAGTGCAGGTCGTAC \\
\hline RT-PAL1-F & CGAACTAGCAGATTGGCAGAGG \\
\hline RT-PAL2-F & GGCGGCGATTGAGAGCAGGA \\
\hline RT-PAL2-R & ATCAGCAGATAGGAAGAGGAGCACC \\
\hline RT-HPPR1-F & TGACTCCAGAAACAACCCACATT \\
\hline RT-HPPR1-R & CCCAGACGACCCTCCACAAG \\
\hline RT-C4H1-F & CCAGGAGTCCAAATAACAGAGCCG \\
\hline RT-C4H1-R & GCCACCAAGCGTTCACCAAGAT \\
\hline RT-4CL1-F & TCACCCATGCCGGATTCGAG \\
\hline RT-4CL1-R & AGATCGCGCCGATGAAGGAG \\
\hline RT-4CL2-F & TCGCCAAATACGACCTTTCC \\
\hline RT-4CL2-R & TGCTTCAGTCATCCCATACCC \\
\hline RT-RAS1-F & CCAAAGTCAATTATGCCAAGGG \\
\hline RT-RAS1-R & GTCGGATAGGTGGTGCTCGT \\
\hline RT-RAS2-F & ACTCGGTTCAAATGCGGTAG \\
\hline RT-RAS2-R & GGGCTGGTATTCGTCGTG \\
\hline RT-CYP98A14-F & CCAATCCTACGGCCCGATCC \\
\hline RT-CYP98A14-R & GCCGTCTCTGCTGAGCTTGA \\
\hline RT-CCR-F & CTGATGTTGCTTCGCCTTCT \\
\hline RT-CCR-R & CATACGTGCCTTCCCCTTG \\
\hline RT-COMT-F & GCCACTAAGAATGTTGTCC \\
\hline RT-COMT-R & TCTGTCCTTTCCTTACCA \\
\hline
\end{tabular}




\subsection{Construction of Transgenic Vectors and Plant Transformation}

The pMDC123SB-AtMIR390a-B/c [58] vector was used to construct the amiRNAmediated miR408-suppressed expression vector. A pair of amiRNA oligonucleotide primers, amiR-MIR408-F/R, were designed using P-SAMS software (http:/ / p-sams.carri ngtonlab.org/, accessed in 25 January 2018). These synthesized amiRNA oligonucleotide primers were annealed, and the formed cassette was then inserted into the pMDC123SBAtMIR390a-B/c vector via BsaI digestion.

For the SmLAC3 overexpression vector, the full-length open reading frame (ORF) was amplified with primers 207-SmLAC3-F/207-SmLAC3-R, which contained attB1/attB2 sites, and was subsequently recombined into the pDONR207 vector by the standard BP reaction. The entry vector pDONR207-SmLAC3 was transferred into the vector pEarleyGate 202 (Earley et al., 2006) by an LR reaction to generate the pEarleyGate 202-SmLAC3 vector, according to the protocol of the Gateway manufacturer (Invitrogen, Carlsbad, USA).

All the recombinant constructs were transformed into S. miltiorrhiza using the Agrobacterium-mediated transformation method [57].

\subsection{Molecular Detection of Transgenic Plantlets}

To detect miR408-suppressed lines and SmLAC3-OE lines at the DNA level, the $2 \times 35 \mathrm{~S}$ CaMV promoter and CaMV35S promoter were amplified, respectively, from genomic DNA, and all the transgenic lines were identified at the DNA level as described previously [59].

The total RNA was isolated from the leaves of two-month-old transgenic plantlets using a Plant RNA Kit (OMEGA, United States). For the expression profiles of SmLAC3, the total RNA was isolated from different plant components (e.g., taproot, lateral root, fibrous root, stem, leaf, corolla, calyx, stamen, and pistil) of two-year-old S. miltiorrhiza at the flowering stage. The RNA was converted to first-strand cDNA with the PrimeScript ${ }^{\mathrm{TM}}$ RT Master Mix (TaKaRa, Beijing, China) according to the manufacturer's protocols. The transcript levels of the enzyme genes and precursor miR408 were analyzed using qRT-PCR, which was performed with the SYBR Premix Ex Taq ${ }^{\mathrm{TM}}$ (TaKaRa, Beijing, China) on a Roche LightCycler ${ }^{\circledR} 96$ System. SmUbiquitin served as the internal reference. The relative expression of the gene was calculated using the comparative $C_{T}$ method [60].

For the transcript level of mature miR408, the reverse transcription of the total RNA was performed using a Mir- $X^{\mathrm{TM}}$ miRNA First-Strand Synthesis Kit (TaKaRa, Beijing, China).

\subsection{RLM-RACE}

The S. miltiorrhiza plantlets under a Cu-deficient condition were used for RNA extraction. The 5' RLM-RACE adapter was obtained through RNA processing following the instruction book. Subsequently, the cDNA acquired by reverse transcription was used as the template for nested PCR according to the protocol of the FirstChoice ${ }^{\circledR}$ RLM-RACE kit (no. AM1700; Invitrogen). The cloned products were inserted into pMD19-T (TaKaRa, Dalian, China) and then sequenced.

\subsection{Determination of Total Phenolic and Total Flavonoid}

The roots of the two-month-old transgenic lines and the control lines were air-dried at $20 \pm 2{ }^{\circ} \mathrm{C}$. The contents of the total phenolic and total flavonoid in the roots were measured according to a previous method [61].

\subsection{Detection of RA and SalB by HPLC}

The levels of RA and SalB were determined from the roots of two-month-old plants. The phenolic acids were extracted and determined using an Agilent ZORBAX SB-C18 column $(250 \times 4.6 \mathrm{~mm}, 5 \mu \mathrm{m})$ on an UltiMate 3000 HPLC System (Thermo Fisher Scientific) equipped with a PDA detector as described previously [61]. 


\subsection{Lignin Detection by Histochemical Staining}

To observe the lignin content changes in different lines, histochemical staining was used to locate and analyze the lignin content. Roots were collected from 60-day-old transgenic lines and control plantlets, and small root segments $(5 \mathrm{~mm}$ long) were cut from the same parts and immersed in a FAA fixation solution for more than 24 hours followed by paraffin embedding. Subsequently, the sections were stained by means of the saffron staining method using the following steps: First, the sections were soaked in a $1 \%$ saffron water solution for about two hours and were rinsed with distilled water and then $75 \%$ ethanol several times, and the slices were sealed with $50 \%$ glycerin. Finally, the processed sections were observed under a visible light microscope.

The air-dried roots of the three-month-old transgenic and control lines were used to determine the lignin content. Contents of Klason lignin (acid-insoluble) and acid-soluble lignin were measured according to a previous protocol [61].

\subsection{Determination of Anthocyanin Concentration}

The leaves of the two-month-old transgenic and control lines were collected and airdried at $20 \pm 2{ }^{\circ} \mathrm{C}$. The anthocyanin concentration was determined according to previous protocols [62].

\section{Conclusions}

Knocking down Sm-miR408 or overexpressing SmLAC3 in S. miltiorrhiza significantly increased the content of SalB and RA. SmLAC3 was verified as the authentic target of SmmiR408. Although the substrate catalyzed by SmLAC3 is unknown, our findings indicated that the miR408-SmLAC3 module participates in SalB synthesis in S. miltiorrhiza.

Author Contributions: Conceptualization, H.Z., X.G., and X.C.; methodology, L.L., R.Y., and J.N.; formal analysis, H.Z., L.L., S.W., and X.C.; investigation, H.Z., X.G., and D.W.; data curation, H.Z., R.Y. and S.W.; writing-original draft preparation, H.Z.; writing-review and editing, X.C.; project administration, J.N., D.W., and X.C. All authors have read and agreed to the published version of the manuscript.

Funding: This research was funded by the National Natural Science Foundation of China (31900254) and the Fundamental Research Funds for the Central Universities (GK202107003).

Conflicts of Interest: The authors declare no conflict of interest.

\section{References}

1. Rogers, K.; Chen, X. Biogenesis, turnover, and mode of action of plant microRNAs. Plant Cell 2013, 25, 2383-2399. [CrossRef]

2. Bartel, D.P. MicroRNAs: Target recognition and regulatory functions. Cell 2009, 136, 215-233. [CrossRef]

3. Chen, X. A microRNA as a translational repressor of APETALA2 in Arabidopsis flower development. Science 2004, 303, $2022-2025$. [CrossRef]

4. Guo, H.S.; Xie, Q.; Fei, J.F.; Chua, N.H. MicroRNA directs mRNA cleavage of the transcription factor NAC1 to downregulate auxin signals for Arabidopsis lateral root development. Plant Cell 2005, 17, 1376-1386. [CrossRef]

5. Aukerman, M.J.; Sakai, H. Regulation of flowering time and floral organ identity by a MicroRNA and its APETALA2-like target genes. Plant Cell 2003, 15, 2730-2741. [CrossRef] [PubMed]

6. Liu, S.; Yang, C.; Wu, L.; Cai, H.; Li, H.; Xu, M. The peu-miR160a-PeARF17.1/PeARF17.2 module participates in the adventitious root development of poplar. Plant Biotechnol. J. 2020, 18, 457-469. [CrossRef]

7. Xue, L.J.; Zhang, J.J.; Xue, H.W. Characterization and expression profiles of miRNAs in rice seeds. Nucleic Acids Res. 2009, 37, 916-930. [CrossRef] [PubMed]

8. Yu, L.; Yu, X.; Shen, R.; He, Y. HYL1 gene maintains venation and polarity of leaves. Planta 2005, 221, 231-242. [CrossRef] [PubMed]

9. Palatnik, J.; Allen, E.; Wu, X.; Schommer, C.; Schwab, R.; Carrington, J.; Weigel, D. Control of leaf morphogenesis by microRNAs. Nature 2003, 425, 257-263. [CrossRef] [PubMed]

10. Zhao, L.; Kim, Y.; Dinh, T.T.; Chen, X. miR172 regulates stem cell fate and defines the inner boundary of APETALA3 and PISTILLATA expression domain in Arabidopsis floral meristems. Plant J. 2007, 51, 840-849. [CrossRef]

11. Khan, G.A.; Declerck, M.; Sorin, C.; Hartmann, C.; Crespi, M.; Lelandais-Briere, C. MicroRNAs as regulators of root development and architecture. Plant Mol. Biol. 2011, 77, 47-58. [CrossRef] [PubMed] 
12. Couzigou, J.M.; Combier, J.P. Plant microRNAs: Key regulators of root architecture and biotic interactions. New Phytol. 2016, 212, 22-35. [CrossRef] [PubMed]

13. Chen, H.; Li, Z.; Xiong, L. A plant microRNA regulates the adaptation of roots to drought stress. FEBS Lett. 2012, 586, 1742-1747. [CrossRef]

14. Sunkar, R.; Kapoor, A.; Zhu, J.-K. Posttranscriptional induction of two Cu/Zn superoxide dismutase genes in Arabidopsis is mediated by downregulation of miR398 and important for oxidative stress tolerance. Plant Cell 2006, 18, 2051-2065. [CrossRef]

15. Abdel-Ghany, S.E.; Pilon, M. MicroRNA-mediated systemic down-regulation of copper protein expression in response to low copper availability in Arabidopsis. J. Biol. Chem. 2008, 283, 15932-15945. [CrossRef]

16. Zhu, C.; Ding, Y.; Liu, H. MiR398 and plant stress responses. Physiol. Plant 2011, 143, 1-9. [CrossRef] [PubMed]

17. Ng, D.W.K.; Zhang, C.; Miller, M.; Palmer, G.; Whiteley, M.; Tholl, D.; Chen, Z.J. Cis- and trans-regulation of miR163 and target genes confers natural variation of secondary metabolites in two Arabidopsis species and their allopolyploids. Plant Cell 2011, 23, 1729-1740. [CrossRef] [PubMed]

18. Gou, J.Y.; Felippes, F.F.; Liu, C.J.; Weigel, D.; Wang, J.W. Negative regulation of anthocyanin biosynthesis in Arabidopsis by a miR156-targeted SPL transcription factor. Plant Cell 2011, 23, 1512-1522. [CrossRef]

19. Zhang, M.; Dong, Y.; Nie, L.; Lu, M.; Fu, C.; Yu, L. High-throughput sequencing reveals miRNA effects on the primary and secondary production properties in long-term subcultured Taxus cells. Front. Plant Sci. 2015, 6, 604. [CrossRef]

20. Zhang, J.P.; Yu, Y.; Feng, Y.Z.; Zhou, Y.F.; Zhang, F.; Yang, Y.W.; Lei, M.Q.; Zhang, Y.C.; Chen, Y.Q. MiR408 regulates grain yield and photosynthesis via a phytocyanin protein. Plant Physiol. 2017, 175, 1175-1185. [CrossRef]

21. Ma, C.; Burd, S.; Lers, A. miR408 is involved in abiotic stress responses in Arabidopsis. Plant J. 2015, 84, 169-187. [CrossRef]

22. Trindade, I.; Capitão, C.; Dalmay, T.; Fevereiro, M.P.; Santos, D.M.d. miR398 and miR408 are up-regulated in response to water deficit in Medicago truncatula. Planta 2009, 231, 705-716. [CrossRef]

23. Pan, J.; Huang, D.; Guo, Z.; Kuang, Z.; Zhang, H.; Xie, X.; Ma, Z.; Gao, S.; Lerdau, M.T.; Chu, C.; et al. Overexpression of microRNA408 enhances photosynthesis, growth, and seed yield in diverse plants. J. Integr. Plant Biol. 2018, 60, 323-340. [CrossRef]

24. Hajyzadeh, M.; Turktas, M.; Khawar, K.M.; Unver, T. miR408 overexpression causes increased drought tolerance in chickpea. Gene 2015, 555, 186-193. [CrossRef]

25. Zhang, H.; He, H.; Wang, X.; Wang, X.; Yang, X.; Li, L.; Deng, X.W. Genome-wide mapping of the HY5-mediated gene networks in Arabidopsis that involve both transcriptional and post-transcriptional regulation. Plant J. 2011, 65, 346-358. [CrossRef]

26. Guo, X.; Niu, J.; Cao, X. Heterologous expression of Salvia miltiorrhiza microRNA408 enhances tolerance to salt stress in Nicotiana benthamiana. Int. J. Mol. Sci. 2018, 19, 3985. [CrossRef] [PubMed]

27. Turlapati, P.V.; Kim, K.-W.; Davin, L.B.; Lewis, N.G. The laccase multigene family in Arabidopsis thaliana: Towards addressing the mystery of their gene function(s). Planta 2010, 233, 439-470. [CrossRef]

28. Cai, X.; Davis, E.J.; Ballif, J.; Liang, M.; Bushman, E.; Haroldsen, V.; Torabinejad, J.; Wu, Y. Mutant identification and characterization of the laccase gene family in Arabidopsis. J. Exp. Bot. 2006, 57, 2563-2569. [CrossRef] [PubMed]

29. Pourcel, L.; Routaboul, J.; Cheynier, V.; Lepiniec, L.; Debeaujon, I. Flavonoid oxidation in plants: From biochemical properties to physiological functions. Trends Plant Sci. 2007, 12, 29-36. [CrossRef] [PubMed]

30. Zhao, Q.; Nakashima, J.; Chen, F.; Yin, Y.; Fu, C.; Yun, J.; Shao, H.; Wang, X.; Wang, Z.-Y.; Dixon, R.A. LACCASE is necessary and nonredundant with PEROXIDASE for lignin polymerization during vascular development in Arabidopsis. Plant Cell 2013, 25, 3976-3987. [CrossRef] [PubMed]

31. Pourcel, L.; Routaboul, J.M.; Kerhoas, L.; Caboche, M.; Lepiniec, L.; Debeaujon, I. TRANSPARENT TESTA10 encodes a laccase-like enzyme involved in oxidative polymerization of flavonoids in Arabidopsis seed coat. Plant Cell 2005, 17, 2966-2980. [CrossRef] [PubMed]

32. Wang, G.D.; Li, Q.J.; Luo, B.; Chen, X.Y. Explanta phytoremediation of trichlorophenol and phenolic allelochemicals via an engineered secretory laccase. Nat. Biotechnol. 2004, 22, 893-897. [CrossRef] [PubMed]

33. Li, Q.; Feng, J.; Chen, L.; Xu, Z.; Zhu, Y.; Wang, Y.; Xiao, Y.; Chen, J.; Zhou, Y.; Tan, H.; et al. Genome-wide identification and characterization of Salvia miltiorrhiza laccases reveal potential targets for salvianolic acid B biosynthesis. Front. Plant Sci. 2019, 10, 435. [CrossRef] [PubMed]

34. Di, P.; Zhang, L.; Chen, J.; Tan, H.; Xiao, Y.; Dong, X.; Zhou, X.; Chen, W. ${ }^{13} \mathrm{C}$ tracer reveals phenolic acids biosynthesis in hairy root cultures of Salvia miltiorrhiza. ACS Chem. Biol. 2013, 8, 1537-1548. [CrossRef]

35. Zhao, M.; Meyers, B.C.; Cai, C.; Xu, W.; Ma, J. Evolutionary patterns and coevolutionary consequences of MIRNA genes and microRNA targets triggered by multiple mechanisms of genomic duplications in soybean. Plant Cell 2015, 27, 546-562. [CrossRef]

36. Voinnet, O. Origin, biogenesis, and activity of plant microRNAs. Cell 2009, 136, 669-687. [CrossRef]

37. Dai, X.; Zhuang, Z.; Zhao, P.X. psRNATarget: A plant small RNA target analysis server (2017 release). Nucleic Acids Res. 2018, 46 , W49-W54. [CrossRef]

38. Zhang, H.; Li, L. SQUAMOSA promoter binding protein-like7 regulated microRNA408 is required for vegetative development in Arabidopsis. Plant J. 2013, 74, 98-109. [CrossRef] [PubMed]

39. Ding, J.; Zhou, S.; Guan, J. Finding microRNA targets in plants: Current status and perspectives. Genom. Proteom. Bioinform. 2012, 10, 264-275. [CrossRef]

40. Llave, C.; Xie, Z.; Kasschau, K.; Carrington, J. Cleavage of scarecrow-like mRNA targets directed by a class of Arabidopsis miRNA. Science 2002, 297, 2053-2056. [CrossRef] 
41. Xiao, Y.; Gao, S.; Di, P.; Chen, J.; Chen, W.; Zhang, L. Methyl jasmonate dramatically enhances the accumulation of phenolic acids in Salvia miltiorrhiza hairy root cultures. Physiol. Plant. 2009, 137, 1-9. [CrossRef] [PubMed]

42. Li, L.; Wang, D.; Zhou, L.; Yu, X.; Yan, X.; Zhang, Q.; Li, B.; Liu, Y.; Zhou, W.; Cao, X.; et al. JA-responsive transcription factor SmMYB97 promotes phenolic acid and tanshinone accumulation in Salvia miltiorrhiza. J. Agric. Food Chem. 2020, 68, 14850-14862. [CrossRef]

43. Wang, J.; Feng, J.; Jia, W.; Chang, S.; Li, S.; Li, Y. Lignin engineering through laccase modification: A promising field for energy plant improvement. Biotechnol. Biofuels 2015, 8, 145. [CrossRef]

44. Liang, M.; Davis, E.; Gardner, D.; Cai, X.; Wu, Y. Involvement of AtLAC15 in lignin synthesis in seeds and in root elongation of Arabidopsis. Planta 2006, 224, 1185-1196. [CrossRef] [PubMed]

45. Petersen, M.; Abdullah, Y.; Benner, J.; Eberle, D.; Gehlen, K.; Hücherig, S.; Janiak, V.; Kim, K.; Sander, M.; Weitzel, C.; et al. Evolution of rosmarinic acid biosynthesis. Phytochemistry 2009, 70, 1663-1679. [CrossRef] [PubMed]

46. Sunkar, R.; Zhu, J.K. Novel and stress-regulated microRNAs and other small RNAs from Arabidopsis. Plant Cell 2004, 16, 2001-2019. [CrossRef]

47. Zhang, F.; Zhang, Y.C.; Zhang, J.P.; Yu, Y.; Zhou, Y.F.; Feng, Y.Z.; Yang, Y.W.; Lei, M.Q.; He, H.; Lian, J.P.; et al. Rice UCL8, a plantacyanin gene targeted by miR408, regulates fertility by controlling pollen tube germination and growth. Rice $2018,11,60$. [CrossRef] [PubMed]

48. Kuo, Y.W.; Lin, J.S.; Li, Y.C.; Jhu, M.Y.; King, Y.C.; Jeng, S.T. MicroR408 regulates defense response upon wounding in sweet potato. J. Exp. Bot. 2019, 70, 469-483. [CrossRef] [PubMed]

49. Janusz, G.; Pawlik, A.; Świderska-Burek, U.; Polak, J.; Sulej, J.; Jarosz-Wilkołazka, A.; Paszczyński, A. Laccase properties, physiological functions, and evolution. Int. J. Mol. Sci. 2020, 21, 966. [CrossRef]

50. Bao, W.; O'malley, D.; Whetten, R.; Sederoff, R. A laccase associated with lignification in loblolly pine xylem. Science 1993, 260, 672-674. [CrossRef]

51. Mayer, A.; Staples, R. Laccase: New functions for an old enzyme. Phytochemistry 2002, 60, 551-565. [CrossRef]

52. Liu, Q.; Luo, L.; Zheng, L. Lignins: Biosynthesis and biological functions in plants. Int. J. Mol. Sci. 2018, 19, 335. [CrossRef]

53. Hu, Q.; Min, L.; Yang, X.; Jin, S.; Zhang, L.; Li, Y.; Ma, Y.; Qi, X.; Li, D.; Liu, H.; et al. Laccase GhLac1 modulates broadspectrum biotic stress tolerance via manipulating phenylpropanoid pathway and jasmonic acid synthesis. Plant Physiol. 2018, 176, 1808-1823. [CrossRef] [PubMed]

54. Wang, X.; Zhuo, C.; Xiao, X.; Wang, X.; Docampo-Palacios, M.; Chen, F.; Dixon, R.A. Substrate specificity of LACCASE8 facilitates polymerization of caffeyl alcohol for C-lignin biosynthesis in the seed coat of Cleome hassleriana. Plant Cell 2020, 32, 3825-3845. [CrossRef] [PubMed]

55. Xu, X.; Chen, X.; Chen, Y.; Zhang, Q.; Su, L.; Chen, X.; Chen, Y.; Zhang, Z.; Lin, Y.; Lai, Z. Genome-wide identification of miRNAs and their targets during early somatic embryogenesis in Dimocarpus longan Lour. Sci. Rep. 2020, 10, 4626. [CrossRef] [PubMed]

56. Li, C.; Li, D.; Zhou, H.; Li, J.; Lu, S. Analysis of the laccase gene family and miR397-/miR408-mediated posttranscriptional regulation in Salvia miltiorrhiza. PeerJ 2019, 7, e7605. [CrossRef] [PubMed]

57. Yan, Y.; Wang, Z. Genetic transformation of the medicinal plant Salvia miltiorrhiza by Agrobacterium tumefaciens-mediated method. Plant Cell Tissue Organ Cult. 2007, 88, 175-184. [CrossRef]

58. Carbonell, A.; Takeda, A.; Fahlgren, N.; Johnson, S.; Cuperus, J.; Carrington, J. New generation of artificial MicroRNA and synthetic trans-acting small interfering RNA vectors for efficient gene silencing in Arabidopsis. Plant Physiol. 2014, 165, 15-29. [CrossRef]

59. Yang, N.; Zhou, W.; Su, J.; Wang, X.; Li, L.; Wang, L.; Cao, X.; Wang, Z. Overexpression of SmMYC2 increases the production of phenolic acids in Salvia miltiorrhiza. Front. Plant Sci. 2017, 8, 1804. [CrossRef]

60. Livak, K.J.; Schmittgen, T.D. Analysis of relative gene expression data using real-time quantitative PCR and the 2(-Delta Delta C(T)) Method. Methods 2001, 25, 402-408. [CrossRef]

61. Zhang, Y.; Yan, Y.; Wang, Z. The Arabidopsis PAP1 transcription factor plays an important role in the enrichment of phenolic acids in Salvia miltiorrhiza. J. Agric. Food Chem. 2010, 58, 12168-12175. [CrossRef] [PubMed]

62. Du, T.; Niu, J.; Su, J.; Li, S.; Guo, X.; Li, L.; Cao, X.; Kang, J. SmbHLH37 functions antagonistically with SmMYC2 in regulating jasmonate-mediated biosynthesis of phenolic acids in Salvia miltiorrhiza. Front. Plant Sci. 2018, 9, 1720. [CrossRef] [PubMed] 